Research Journal of Applied Sciences 6 (1): 53-60, 2011

ISSN: $1815-932 \mathrm{X}$

(C) Medwell Journals, 2011

\title{
The Effects of Nonlinear Gain and Thermal Carrier Escape on Dynamic Characterizations of GaAs/InGaAs Self-Assembled Quantum Dot Lasers
}

\author{
H. Arabshahi and D. Ghodsi Nahri \\ Department of Physics, Mashhad Branch, Islamic Azad University, Mashhad, Iran
}

\begin{abstract}
In this study researchers have studied the effects of nonlinear gain and thermal carrier escape on dynamic characteristics of GaAs/InGaAs self-assembled quantum dot laser with considering the homogeneous and inhomogeneous broadening of the optical gain using fourth order Runge-Kutta method. The calculations show that the thermal carrier escape leads to shift the dominant lasing mode at the low injection currents. The number of lasing modes increases for the larger injection currents. With exceeding the FWHM of homogeneous broadening from the full width at half maximum FWHM of inhomogeneous broadening, the dynamic and static characteristics degrade and SAQD-LD reaches the steady-state slower. The threshold current, the steady-state photons and the dynamic-characteristics degrade and SAQD-LD reaches the steady-state slower as the FWHM of inhomogeneous broadening and carrier relaxation life time increase.
\end{abstract}

Key words: Nonlinear gain, self-assembled quantum dot laser, Runge-Kutta method, thermal carrier escape, homegeneous, inhomogeneous

\section{INTRODUCTION}

Three-dimensional quantum confinement of electrons, holes and excitons in semiconductor microstructures known as quantum dots is predicted to produce new physical phenomena and improve optoelectronic devices significantly (Zhang et al., 2008; Liu et al., 2005; Lv et al., 2008). The atom like state density in quantum dots associated with three dimensional confinement of electrons and holes would cause an increase of optical gain and limit thermal carrier distribution

Therefore, the use of quantum dots for semiconductor lasers as an active region is expected to provide a remarkable reduction of threshold current and temperature sensitivity. During primary research based on predictions in the early 1980 s, quantum dots have been created by combining lithography and re-growth on a processed substrate.

The artificial techniques however, suffer from non-uniform doe size, poor interface quality and low numerical density. High uniformity is required to achieve the atom-like state density of a dot ensemble. A high optical quality and high numerical density are required to obtain a large gain. Quantum dot lasers using artificial techniques therefore, showed a high threshold current density (Sugawara, 1999; Sugawara et al., 2000). In this study, considering the homogeneous and inhomogeneous broadening of the optical gain also without and with considering the nonlinear gain and thermal escape of carriers from QDs, researchers solve the rate equations numerically using fourth-order RungeKutta method and analyze the dynamics characteristics of GaAs/InGaAs SAQD-LDs.

We show that considering the nonlinear gain result in the dynamic-characteristic of photons number at the FWHM of homogeneous broadening comparable, near or equal to the FWHM of inhomogeneous broadening reaches the steady-state faster.

The dynamics characteristics such as maximum of the relaxation oscillation magnitude, turn-on delay, relaxation oscillation frequency and modulation bandwidth also the steady-state photons number improve as the current increases. Threshold current, turn-on delay and steady-state photons number increase and relaxation oscillation frequency decreases for larger FWHM of homogeneous broadening.

\section{LINEAR AND NONLINEAR OPTICAL GAIN}

Based on the density-matrix theory, the linear optical gain of QD active region is given as Bilenca and Eisenstein (2004):

$$
g^{(1)}(E)=\frac{2 \mathrm{pe}^{2} h N_{D}}{\mathrm{cn}_{\mathrm{r}} \mathrm{e}_{0} \mathrm{~m}_{0}^{2}} \times \frac{\left|\mathrm{p}_{\mathrm{cv}}^{\mathrm{s}}\right|\left(\mathrm{f}_{\mathrm{c}}-\mathrm{f}_{\mathrm{v}}\right)}{\mathrm{E}_{\mathrm{cv}}} \mathrm{B}_{\mathrm{cv}}\left(\mathrm{E}-\mathrm{E}_{\mathrm{cv}}\right)
$$

Corresponding Author: H. Arabshahi, Department of Physics, Mashhad Branch, Islamic Azad University, Mashhad, Iran 
Where:

$\mathrm{n}_{\mathrm{r}} \quad=$ The refractive index

$\mathrm{N}_{\mathrm{D}}=$ The volume density of QDs

$\left|\mathrm{P}_{\mathrm{cv}}^{\sigma}\right|^{2}=$ The transition matrix element

$\mathrm{f}_{\mathrm{c}}=$ The electron occupation function of the conduction-band discrete state

$\mathrm{f}_{\mathrm{v}}=$ That of the valence-band discrete state

$\mathrm{E}_{\mathrm{CV}}=$ The interband transition energy

The linear optical gain shows the homogeneous broadening of a Lorentz shape as Bilenca and Eisenstein (2004):

$$
\mathrm{B}_{\mathrm{cv}}\left(\mathrm{E}-\mathrm{E}_{\mathrm{cv}}\right)=\frac{\mathrm{hG}_{\mathrm{cv}} / \mathrm{p}}{\left(\mathrm{E}-\mathrm{E}_{\mathrm{cv}}\right)^{2}+\left(\mathrm{hG}_{\mathrm{cv}}\right)^{2}}
$$

Where FWHM is given as $2 \hbar \Gamma_{c y}$ with polarization dephasing or scattering rate $\Gamma_{\mathrm{cv}}$. Neglecting the optical-field polarization dependence, the transition matrix element is given as:

$$
\left|P_{c v}^{s}\right|^{2}=\left|I_{c v}\right|^{2} M^{2}
$$

where, $I_{c v}$ represents the overlap integral between the envelope functions of an electron and a hole:

$$
\mathrm{M}^{2}=\frac{\mathrm{m}_{0}^{2}}{12 \mathrm{~m}_{\mathrm{e}}^{*}} \times \frac{\mathrm{E}_{\mathrm{g}}\left(\mathrm{E}_{\mathrm{g}}+\mathrm{D}\right)}{\mathrm{E}_{\mathrm{g}}+2 \mathrm{D} / 3}
$$

Where:

$\mathrm{k} \cdot \mathrm{p}=$ The interaction between the conduction band and valence band

$\mathrm{E}_{\mathrm{g}}=$ The band gap

$\mathrm{m}_{\mathrm{e}}^{*}=$ The electron effective mass

$\mathrm{E}_{\mathrm{g}}=$ The spin-orbit interaction energy of the QD material

Equation 3 holds as long as we consider QDs with a nearly symmetrical shape (Tan et al., 2007, 2008). In actual SAQD-LDs, we should rewrite the linear optical gain formula of Eq. 1 by taking into account inhomogeneous broadening due to the QD size and composition fluctuation in terms of a convolution integral as:

$$
\begin{aligned}
& \mathrm{g}^{(1)}(\mathrm{E})=\frac{2 \mathrm{pe}^{2} h N_{D}}{\mathrm{cn}_{\mathrm{r}} \mathrm{e}_{0}^{2}} \cdot \frac{\left|\mathrm{p}_{\mathrm{cv}}^{\mathrm{s}}\right|^{2}}{\mathrm{E}_{\mathrm{cv}}}\left(\mathrm{f}_{\mathrm{c}}(\mathrm{E})-\mathrm{f}_{\mathrm{v}}(\mathrm{E})\right) . \\
& \mathrm{B}_{\mathrm{cv}}\left(\mathrm{E}-\mathrm{E}_{\mathrm{c}}\right) \mathrm{G}\left(\mathrm{E}_{\mathrm{c}}-\mathrm{E}_{\mathrm{cv}}\right) \mathrm{dE}_{\mathrm{c}}
\end{aligned}
$$

Where:

$\mathrm{E}_{\mathrm{cv}}=$ The center of the energy distribution function of each interband transition
$f_{c}\left(E_{c}\right)=$ The electron occupation function of the conduction-band discrete state of the QDs with the interband transition energy of $\mathrm{E}_{\mathrm{c}}$

$f_{v}\left(E_{v}\right)=$ That of the valence band discrete state

The energy fluctuation of QDs are represented by G $\left(\mathrm{E}_{\mathrm{c}}-\mathrm{E}_{\mathrm{cv}}\right)$ that takes a Gaussian distribution function as:

$$
\mathrm{G}\left(\mathrm{E}_{\mathrm{c}}-\mathrm{E}_{\mathrm{cv}}\right)=\frac{1}{\sqrt{2 \mathrm{p} \mathrm{x}_{0}}} \exp \left(\mathrm{E}_{\mathrm{c}}-\mathrm{E}_{\mathrm{cv}}\right)^{2} / 2 \mathrm{x}_{0}^{2}
$$

Whose FWHM is given by $\Gamma_{0}=2.35 \xi_{0}$. The width $\Gamma_{0}$ usually depends on the band index $\mathrm{c}$ and $\mathrm{v}$ (Sugawara et al., 2000).

\section{RATE EQUATIONS}

The most popular and useful way to deal with carrier and photon dynamics in lasers is to solve rate equations for carrier and photons (Markus et al., 2003; Grundmann, 2002). Researchers consider an electron and a hole as an exciton, thus, the relaxation means the process that both an electron and a hole relax into the ground state simultaneously to form an exciton. We assume that only a single discrete electron and hole ground state is formed inside the QD and the charge neutrality always holds in each QD.

In order to describe the interaction between the QDs with different resonant energies through photons, we divide the $\mathrm{QD}$ ensemble into $\mathrm{j}=1,2, \ldots 2 \mathrm{M}+1$ groups, depending on their resonant energy for the interband transition over the longitudinal cavity photon modes. $j=m$ corresponds to the group and mode at $E_{c v}$. We take the energy width of each group equal to the mode separation of the longitudinal cavity photon modes which equals to:

$$
\mathrm{D}_{\mathrm{E}}=\mathrm{ch} / 2 \mathrm{n}_{\mathrm{r}} \mathrm{L}_{\mathrm{ca}}
$$

where, $\mathrm{L}_{\mathrm{ca}}$ is the cavity length. The energy of the jth QDs group is represented by:

$$
E_{j}=E_{c v}-(M-j) D_{E}
$$

Where $\mathrm{j}=1,2, \ldots, 2 \mathrm{M}+1$. The QD density jth QDs group is given as:

$$
N_{D} G_{j}=N_{D} G\left(E_{j}-E_{c V}\right) D_{E}
$$

Let $\mathrm{N}_{\mathrm{j}}$ be the carrier number in $\mathrm{jth}$ QDs group according to Pauli's exclusion principle, the occupation probability in the ground state of the $\mathrm{jth}$ QDs group is defined as: 


$$
P_{j}=N_{j} / 2 N_{D} V_{a} G_{j}
$$

The rate equations are as follows (Tan et al., 2008)

$$
\begin{gathered}
\frac{d N_{s}}{d t}=\frac{I}{e}-\frac{N_{s}}{\tau_{s}}-\frac{N_{s}}{\tau_{s r}}+\frac{N_{w}}{\tau_{w e}} \\
\frac{d N_{w}}{d t}=\frac{N_{s}}{\tau_{s}}+\frac{N_{j}}{\tau_{\mathrm{e}} D_{g}}-\frac{N_{w}}{\tau_{w r}}-\frac{N_{w}}{\tau_{w e}}-\frac{N_{w}}{\tau_{d}} \\
\frac{d N j}{d t}=\frac{N_{w} G_{j}}{\tau_{d j}}-\frac{N_{j}}{\tau_{r}}-\frac{N_{j}}{\tau_{e} D_{j}}-\frac{c \Gamma}{n_{r}} \cdot g^{(1)}(E) S_{m} \\
\frac{d S m}{d t}=\frac{\beta N_{j}}{\tau_{r}}+\frac{c \Gamma}{n_{r}} \cdot g^{(1)}(E) S_{m}-S_{m} / \tau_{p}
\end{gathered}
$$

where, $N_{S}, N_{w}$ and $N_{j}$ are the carrier number in Separate Confinement Heterostructure (SCH) layer, Wetting Layer (WL) and jth QDs group, respectively, $\mathrm{S}_{\mathrm{m}}$ is the photon number of mth mode where $\mathrm{m}=1,2 \ldots .2 \mathrm{M}+1$, I is the injected current, $G_{j}$ is the fraction of the jth QDs group type within an ensemble of different dot size populations, $\mathrm{e}$ is the electron charge, $\mathrm{D}_{\mathrm{g}}$ is the degeneracy of the QD ground state without spin, $\beta$ is the spontaneous-emission coupling efficiency to the lasing mode. $\mathrm{g}_{\mathrm{m}}{ }^{(1)}$ is the linear optical gain which the jth QDs group gives to the mth mode photons where is represented by:

$$
\begin{aligned}
& \mathrm{g}_{\mathrm{mj}}^{(1)}(\mathrm{E})=\frac{2 \pi \mathrm{e}^{2} h N_{D}}{\mathrm{cn}_{\mathrm{r}} \varepsilon_{0} \mathrm{e}_{0}^{2}} \cdot \frac{\left|\mathrm{p}_{\mathrm{cv}}^{\mathrm{s}}\right|^{2}}{\mathrm{E}_{\mathrm{cv}}}\left(2 \mathrm{p}_{\mathrm{j}}-1\right) . \\
& \mathrm{G}_{\mathrm{j}} \mathrm{B}_{\mathrm{cv}}\left(\mathrm{E}_{\mathrm{m}}-\mathrm{E}_{\mathrm{j}}\right)
\end{aligned}
$$

The related time constants are as $\tau_{s}$, diffusion in the $\mathrm{SCH}$ region, $\tau_{\mathrm{sr}}$ carrier recombination in the $\mathrm{SCH}$ region, $\tau_{\mathrm{w} \theta}$ carrier reexcitation from the WL to the SCH region, $\tau_{\mathrm{wr}}$, carrier recombination in the $\mathrm{WL}, \tau_{\text {dij }}$, carrier relaxation into the jth QDs group, $\tau_{\mathrm{p}}$ carrier recombination in the QDs, $\tau_{\mathrm{p}}$, photon lifetime in the cavity, The average carrier relaxation lifetime, $\bar{\tau}_{\mathrm{d}}$ is given as:

$$
\tau_{\mathrm{d}}^{-1}=\tau_{\mathrm{dh}}^{-1} \cdot \mathrm{G}_{\mathrm{n}}=\tau_{\mathrm{d}}^{-1}\left(1-\mathrm{P}_{\mathrm{n}}\right) \mathrm{G}_{\mathrm{n}}
$$

where, $\tau_{0}$ is the initial carrier relaxation lifetime. The photon lifetime in the cavity is:

$$
\tau_{\mathrm{p}}^{-1}=\mathrm{c} / \mathrm{n}_{\mathrm{r}}+\ln \left(1 / \mathrm{R}_{1} \mathrm{R}_{2}\right) / 2 \mathrm{~L}_{\mathrm{cav}}
$$

where, $R_{1}$ and $R_{2}$ are the cavity mirror reflectivity and $\alpha_{1}$ is the internal loss. The laser output power of the mth mode from one cavity mirror is given a:

$$
\mathrm{I}_{\mathrm{m}}=\mathrm{hw}_{\mathrm{m}} \mathrm{cS}_{\mathrm{m}} \ln (1 / \mathrm{R}) / 2 \mathrm{~L}_{\mathrm{cax}} \mathrm{n}_{\mathrm{r}}
$$

where, $\mathrm{w}_{\mathrm{m}}$ is the emitted photon frequency and $\mathrm{R}$ is $\mathrm{R}_{1}$ or $\mathrm{R}_{2}$. We solved the rate equations numerically using fourth order Runge-Kutta method (Sugawara, 1995) to obtain the carrier and photon characteristics and modulation response by supplying the steplike current at time $\mathrm{t}=0$.

The system reaches the steady-state after the relaxation oscillation. We assume that all the carriers are injected into the WL, i.e., $\tau_{\mathrm{sr}}=\tau_{\mathrm{we}}=\infty$ and consider the thermal carrier escape time $\tau_{e}=\infty$.

\section{ANALYSIS OF THE EFFECTS OF NONLINEAR GAIN AND THERMAL CARRIER ESCAPE ON DYNAMIC CHARACTERISTICS}

We simulated previous characteristics with considering the nonlinear gain and thermal carrier escape. The simulation results are similar to previous results except to follow cases. Figure 1 shows photon characteristics for different injection currents $\mathrm{I}=2,2.5,5$ and $10 \mathrm{~mA}$ when the FWHM of homogeneous considering the nonlinear gain and thermal carrier escape from QDs.

As shown in Fig. 1a, the lasing photons at $I=5$ and $10 \mathrm{~mA}$ reach the steady-state after 40 and $30 \mathrm{nsec}$. It is also seen from Fig. $1 \mathrm{~b}$ that the lasing photons at $\mathrm{I}=5$ and $10 \mathrm{~mA}$ do not reach lesser than that of $\mathrm{I}=2 \mathrm{~mA}$ and reach the steady-state after 80 and 70 nsec. Lasing photons at $\mathrm{I}=10 \mathrm{~mA}$ do not reach lesser than that of $\mathrm{I}=$ $5 \mathrm{~mA}$ and the lasing photons at $\mathrm{I}=2.5 \mathrm{~mA}$ reach the steady-state after 60 nsec.

As shown in Fig. 1c, the lasing photons at $I=5$ and $10 \mathrm{~mA}$ after than 20 and $10 \mathrm{~ns}$ and that of $\mathrm{I}=2.5 \mathrm{~mA}$ after $30 \mathrm{nsec}$ reach the steady-state. Also it is shown in Fig. 1d that the dynamic-characteristics of the RoomTemperature (RT) operation of SAQD-LDs, the lasing photons at $\mathrm{I}=5$ and $10 \mathrm{~mA}$ reach the steady-state after 40 and 30 nsec.

These non steady-states, unlike previous case that we do not consider the nonlinear gain and thermal carrier escape, decreases with increasing the injection current. Figure 2 shows RT photon-characteristics for the central lasing mode and close modes around it at currents $\mathrm{I}=2.1$, $2.15,5$ and $10 \mathrm{~mA}$.

As shown in Fig. 2a, maximum of the lasing photons are relate to the mode $\mathrm{M}-1$. This shift of dominant lasing mode is due to the thermal carrier escape effect which it wastes with increasing the injection current (Fig. $2 \mathrm{c}$ and d). Researchers see that the lasing photons at the small 
Res. J. Applied Sci., 6 (1): 53-60, 2011
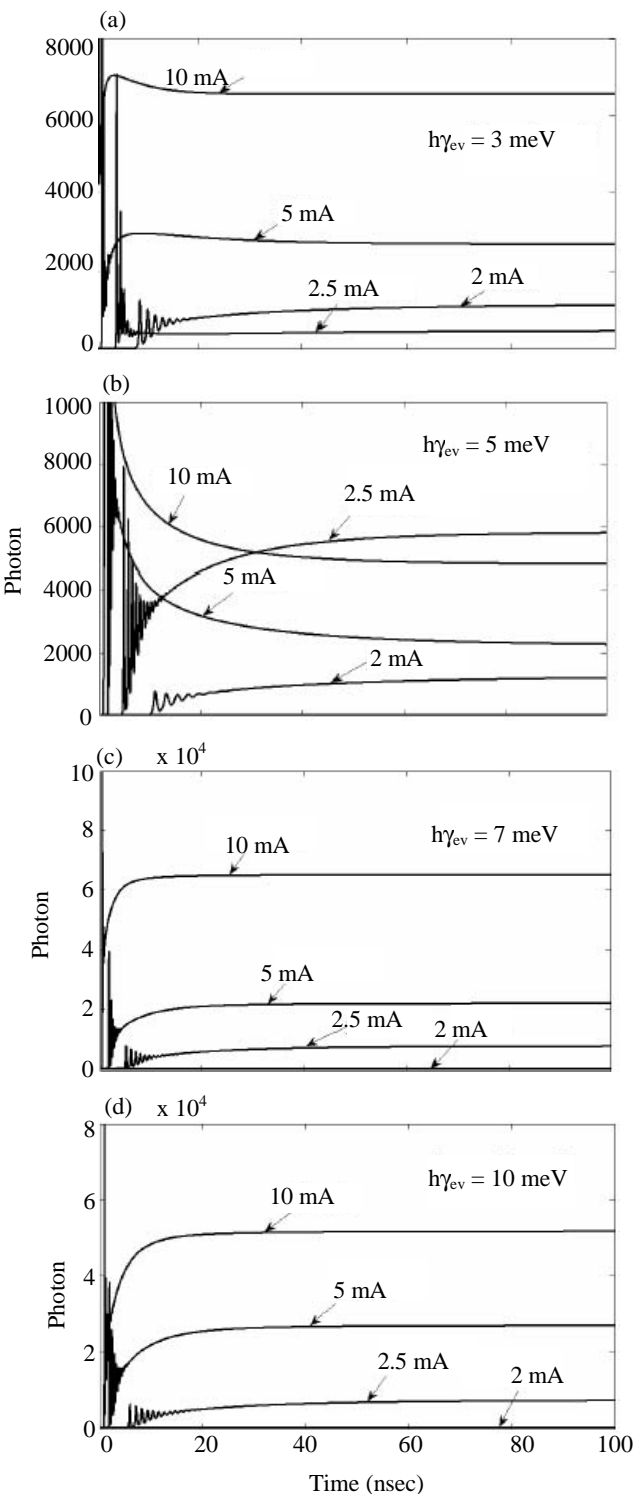

Fig. 1: Photon-characteristics for different injection currents $\mathrm{I}=2,2.5,5$ and $10 \mathrm{~mA}$ when the FWHM of homogeneous broadening is (a) $3 \mathrm{meV}$, (b) 5 $\mathrm{meV}$, (c) $7 \mathrm{meV}$ and (d) $10 \mathrm{meV}$ with considering the nonlinear gain and thermal carrier escape from Qds

FWHM of homogeneous broadening that all of the different QD groups having an optical gain larger than threshold gain laze independently or nearly independently, reach the steady-state after finishing the relaxation oscillation but at the FWHM of homogeneous broadening comparable, near or equal to the FWHM of inhomogeneous broadening without considering the nonlinear gain, the central mode photons, at the time of relaxation oscillation and after finishing it, receive gain not

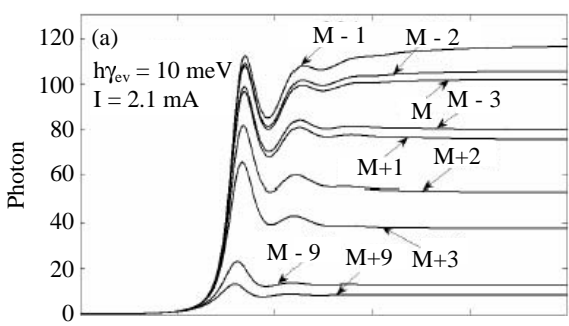

(b)
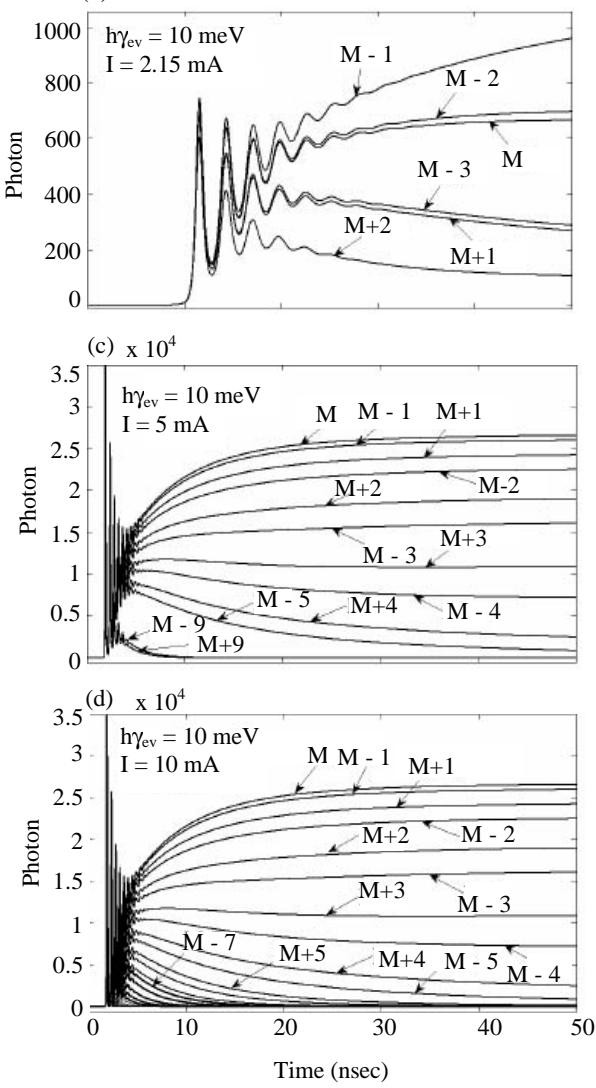

Fig. 2: RT photon-characteristics for the central lasing mode and close modes around it at currents $\mathrm{I}=2.1,2.15,5$ and $10 \mathrm{~mA}$

only from the central group of QDs but also from more or all the groups infinitely, since carriers of these groups do intra band relaxation and put into the central lasing mode by the stimulated emission as a result, lasing emission with one line occurs (Bilenca and Eisenstein, 2004) and the central mode photons increase infinitely. When we consider the total gain at the FWHM of homogeneous broadening near or equal to the FWHM of inhomogeneous broadening, the central mode photons at the time of relaxation oscillation and after finishing it, increase and after several nanoseconds reach the steady-state. This is because, the carriers of QDs group which lie within the FWHM of inhomogeneous 
Res. J. Applied Sci., 6 (1): 53-60, 2011
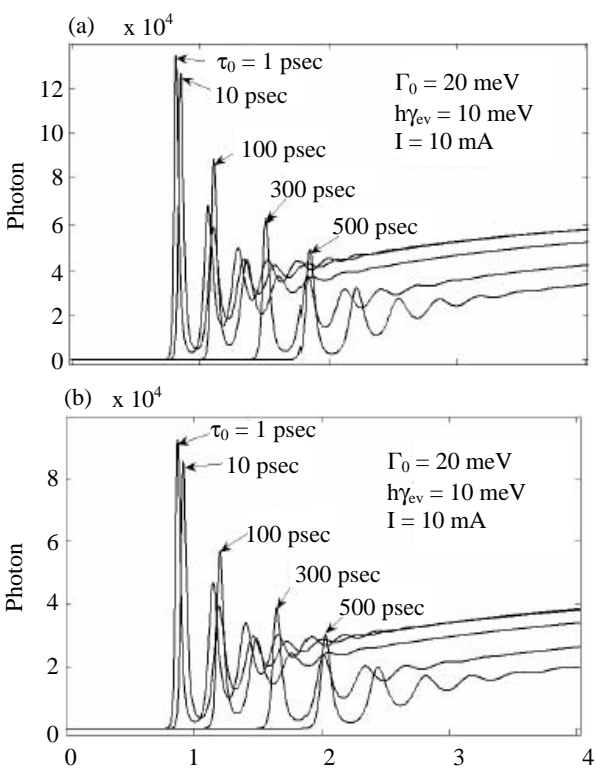

Fig. 3: Simulation results for the RT lasing at different initial carrier relaxation lifetimes $1,10,100,300$ and $500 \mathrm{psec}$ at (a) $10 \mathrm{meV}$ and (b) $20 \mathrm{meV}$

broadening of the central mode, do intra band relaxation and emit into the central lasing mode and close modes around it, the number of these modes and value of emitting into them increase with increasing the injection current. This emission becomes fixing after several nanoseconds due to the gain saturation. As a result, lasing emission with multimode occurs. At the FWHM of homogeneous broadening comparable to the FWHM of inhomogeneous broadening, $3 \mathrm{meV}$ at the injection currents $\mathrm{I}=2.5,5$ and $10 \mathrm{~mA}$ and $5 \mathrm{meV}$ at the injection currents $\mathrm{I}=5$ and $10 \mathrm{~mA}$, the central mode photons decrease due to emitting the central group carriers into other modes (Fig. 1a and b).

They becomes fixing after several nanoseconds as result of the gain saturation at those modes. We treat the effects of the carrier relaxation lifetime and inhomogeneous broadening on dynamic characteristics. Figure 3 shows the simulation results of photon characteristics with considering the nonlinear gain and thermal carrier escape, for the RT lasing at different initial carrier relaxation lifetimes of $1,10,100,300$ and $500 \mathrm{psec}$ at the FWHM of inhomogeneous broadenings $10 \mathrm{meV}$ and $20 \mathrm{meV}$. As shown in Fig. 4 with increasing the carrier relaxation lifetime, maximum of the relaxation oscillation magnitude and the steady-state photons decrease, turnon delay also increases. Turn-on delay increases as the inhomogeneous broadening increases because both of central group and other groups DOS decrease as carriers
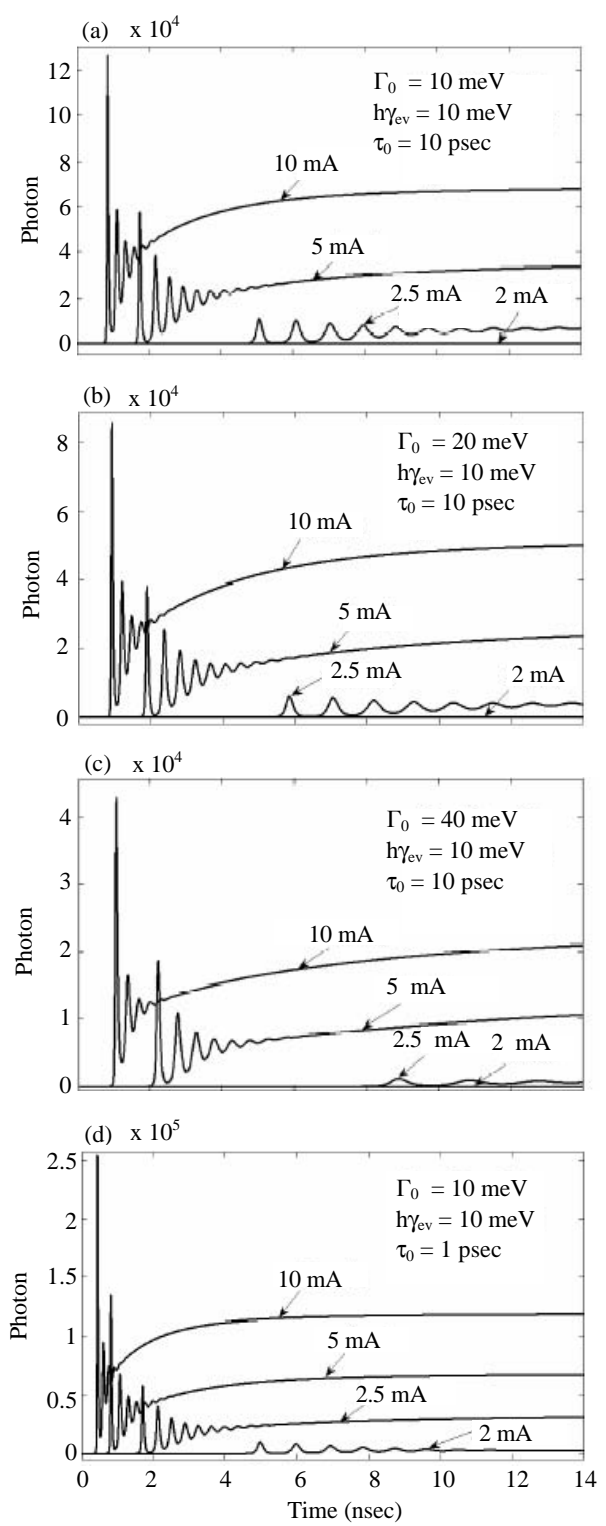

Fig. 4: Calculated results for the FWHM of inhomogeneous broadenings (a) $10 \mathrm{meV}$, (b) 20 $\mathrm{meV}$ and (c) $40 \mathrm{meV}$ at the initial carrier relaxation lifetime $10 \mathrm{psec}$ and (d) $10 \mathrm{meV}$ at the initial carrier relaxation life time $1 \mathrm{psec}$ for different injection currents $\mathrm{I}=2,2.5,5$ and $10 \mathrm{~mA}$

that emit into the central mode decrease more than decreasing the number of states of the central group. Figure 5 shows calculated results for the FWHM of inhomogeneous broadenings (a) $10 \mathrm{meV}$, (b) $20 \mathrm{meV}$ and (c) $40 \mathrm{meV}$ at the initial carrier relaxation lifetime $10 \mathrm{psec}$ and (d) $10 \mathrm{meV}$ at the initial carrier relaxation lifetime 1 psec for different injection currents $\mathrm{I}=2,2.5,5$ and $10 \mathrm{~mA}$. As shown in Fig. 4 with increasing the inhomogeneous 
Res. J. Applied Sci., 6 (1): 53-60, 2011

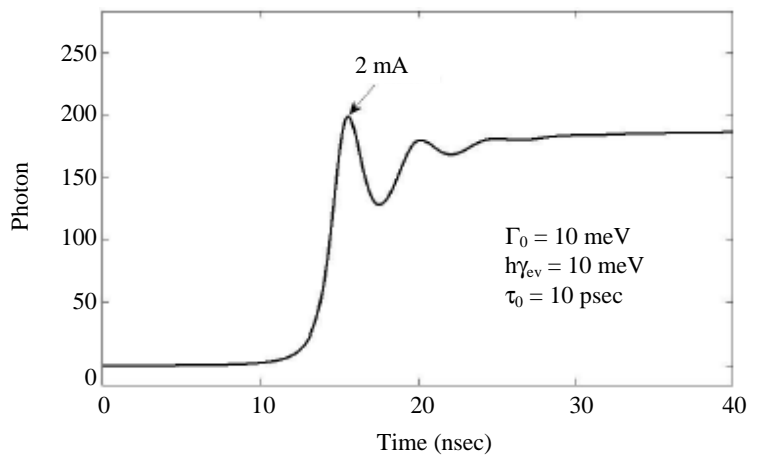

Fig. 5: Lasing photons at the injection current $2 \mathrm{~mA}$ for the FWHM of inhomogeneous broadening $10 \mathrm{meV}$

broadening, the maximum of the relaxation oscillation magnitude and the steady-state photons decrease, laser reaches the steady-state slower. The threshold current also increases because the carriers that emit into the central mode decrease more than decreasing the number of states of the central group. Figure 4 shows the lasing photons at the injection current $\mathrm{I}=2 \mathrm{~mA}$ for the FWHM of inhomogeneous broadening $10 \mathrm{meV}$.

As shown in Fig. 5, there is the lasing emission unlike Fig. 3b. Comparing Fig. 3a and 3d, the threshold current increases and the relaxation oscillation frequency decreases, laser also reaches the steady-state slower as the carrier relaxation lifetime increases due to increasing consumption of carriers in WL.

Figure 6 shows the photon-characteristics at the FWHM of homogeneous broadenings (a) $10 \mathrm{meV}$, (b) 15 $\mathrm{meV}$, (c) $20 \mathrm{meV}$ and (d) $30 \mathrm{meV}$ and injection currents I = 2, 2.5, 5 and $10 \mathrm{~mA}$ for the FWHM of inhomogeneous broadening $20 \mathrm{meV}$.

As shown in Fig. 6 with exceeding the FWHM of homogeneous broadening from the FWHM of inhomogeneous broadening, maximum of the relaxation oscillation magnitude and the steady-state photons decrease, turn-on delay and the threshold current increase. Relaxation oscillation frequency until the FWHM of homogeneous broadening $40 \mathrm{meV}$ decreases and then increases, laser also reaches the steady-state slower.

As a result with exceeding the homogeneous broadening from the inhomogeneous broadening, the dynamic and static-characteristics of SAQD-LD degrade due to increasing the empty DOS. We also simulated the modulation response of GaAs/InGaAs SAQD-LD with considering the nonlinear gain and thermal carrier escape. Figure 7 shows the simulation results for the RT lasing at different injection currents $2.5,5,7.5,12.5$.
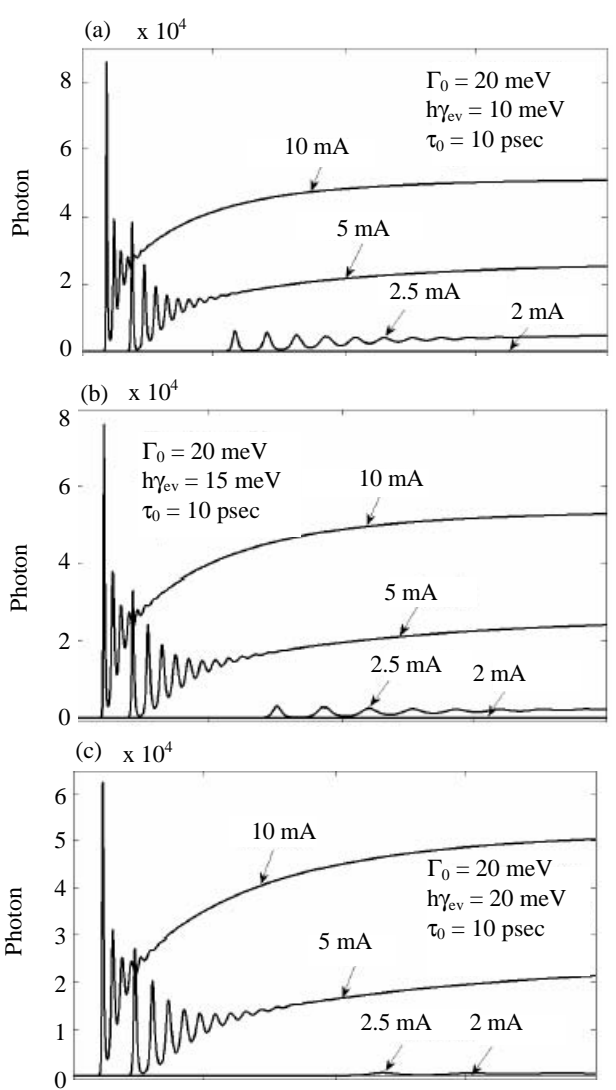

(d) $\times 10^{4}$

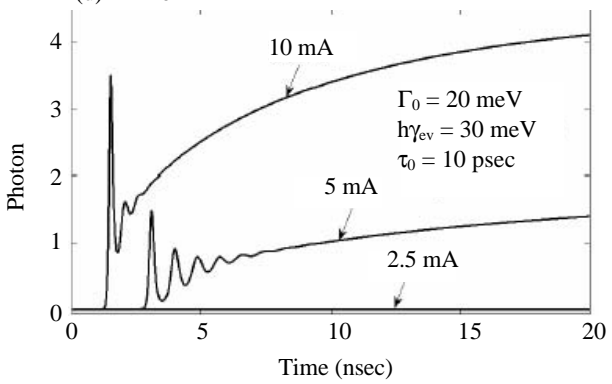

Fig. 6: Photon-characteristics at the FWHM of homogeneous broadenings (a) $10 \mathrm{meV}$ (b) $15 \mathrm{meV}$, (c) $20 \mathrm{meV}$ and (d) $30 \mathrm{meV}$ and injection currents $\mathrm{I}=2,2.5,5$ and $10 \mathrm{~mA}$ for the FWHM of inhomogeneous broadening $20 \mathrm{meV}$

As shown in Fig. 7 modulation bandwidth and relaxation oscillation frequency increase as the injection current increases. This is due to the increase of carriers in central group of Qds.

Figure 8 also shows the modulation response at the injection currents (a) $\mathrm{I}=8$ and (b) $\mathrm{I}=10 \mathrm{~mA}$ when the FWHM of homogeneous broadening is $0.1,3,5$ and $10 \mathrm{meV}$. As the FWHM of homogeneous broadening increases, relaxation oscillation frequency and modulation bandwidth decrease. Modulation response amplitude until 


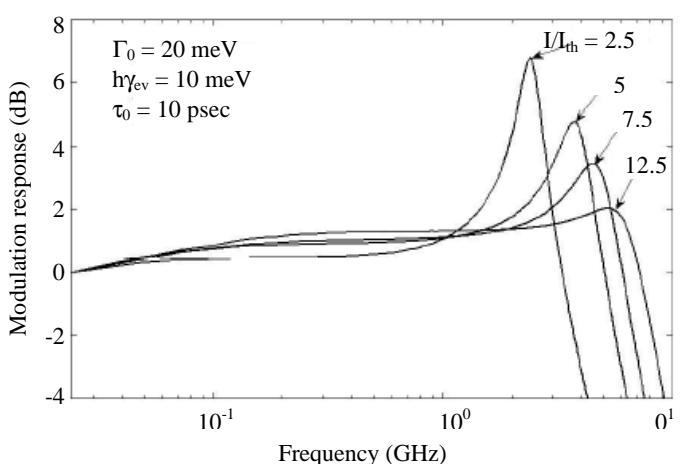

Fig. 7: Calculated modulation response of the RT lasing for different injection currents $2.5,5,7.5,12.5$
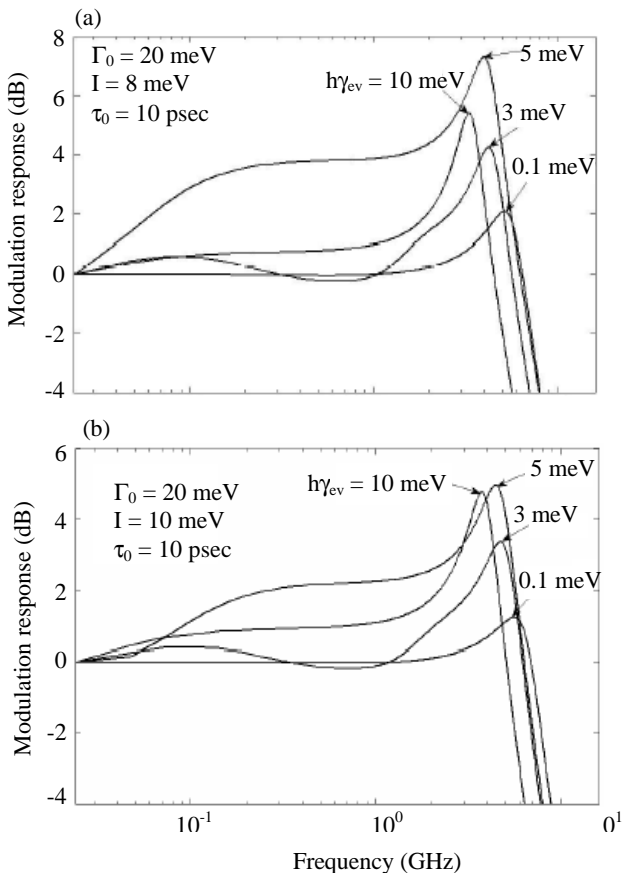

Fig. 8: Modulation response at (a) $\mathrm{I}=5 \mathrm{~mA}$ and (b) $\mathrm{I}=10$ $\mathrm{mA}$ when the FWHM of homogeneous broadening is $0.1,3,5$ and $10 \mathrm{meV}$

the FWHM of homogeneous broadening $10 \mathrm{meV}$ decreases and then increases. Figure 9a shows the modulation response of the RT lasing for different FWHM of inhomogeneous broadenings 10,20 and $40 \mathrm{meV}$. Relaxation oscillation frequency, modulation bandwidth, modulation response amplitude decrease as the FWHM of inhomogeneous broadening increases.

Figure $9 \mathrm{~b}$ also shows the modulation response of the RT lasing for different initial carrier relaxation lifetimes 1 , $10,25,50$ and 100 psec.

Relaxation oscillation frequency, modulation bandwidth and modulation response amplitude decrease
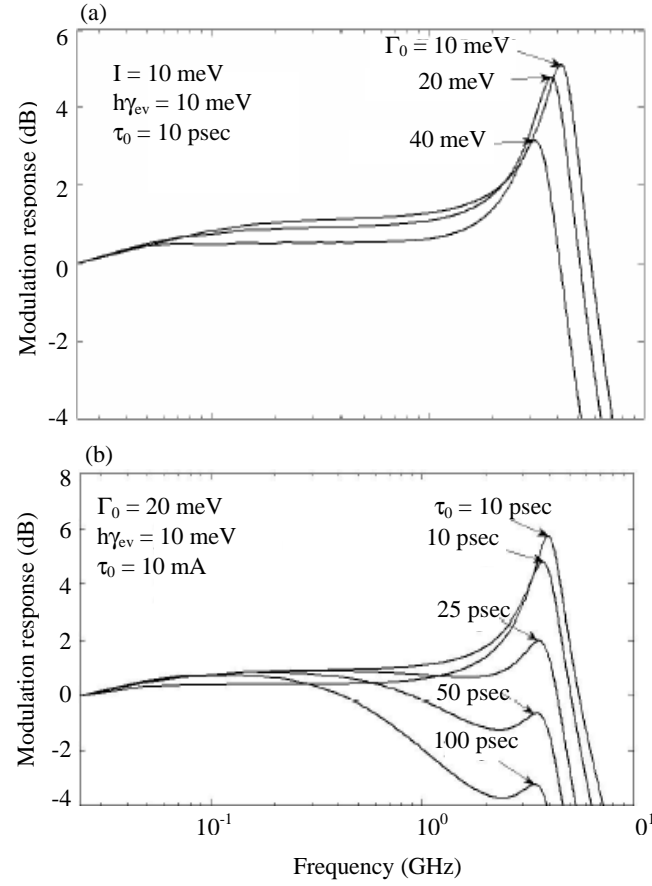

Fig. 9: Modulation response of the RT lasing for different (a) FWHM of inhomogeneous broadenings 10, 20 and $40 \mathrm{meV}$ and (b) initial carrier relaxation lifetimes $1,10,25,50$ and $100 \mathrm{psec}$

as the carrier relaxation lifetime increases. Modulation response amplitude at the relaxation oscillation frequency for 100 psec reduces to $-3.241 \mathrm{~dB}$.

\section{CONCLUSION}

Self-assembled Quantum Dots (QDs) with broadband emitting spectra, QD Super Luminescent Diodes (SLDs) and external cavity tunable QD laser have been studied. The calculations show that the thermal carrier escape leads to shift the dominant lasing mode at the low injection currents. The number of lasing modes increases for the larger injection currents. With exceeding the FWHM of homogeneous broadening from the full width at half maximum FWHM of inhomogeneous broadening, the dynamic and static-characteristics degrade and SAQD-LD reaches the steady-state slower. The threshold current, the steady-state photons and the dynamiccharacteristics degrade and SAQD-LD reaches the steady-state slower as the FWHM of inhomogeneous broadening and carrier relaxation life time increase.

\section{ACKNOWLEDGMENT}

The researchers would like to thank Maryam Gholvani for writing up the study. 


\section{REFERENCES}

Bilenca, A. and G. Eisenstein, 2004. On the noise properties of linear and nonlinear quantum-dot semiconductor optical amplifiers: The impact of inhomogeneously broadened gain and fast carrier dynamics. IEEE J. Quantum Electron., 40: 690-702.

Grundmann, M., 2002. Nano-Optoelectronics: Concepts, Physics and Devices. 1st Edn., Springer-Verlag Berlin, pp: 442.

Liu, N., P. Jin and Z.G. Wang, 2005. InAs/GaAs quantumdot super luminescent diodes with $110 \mathrm{~nm}$ bandwidth. Electron. Lett., 41: 1400-1402.

Lv, X.Q., N. Liu, P. Jin and Z.G. Wang, 2008. Broadband emitting super luminescent diodes with InAs quantum dots in AlGaAs matrix. IEEE Photon. Technol. Lett., 20: 1742-1744.

Markus, A., J.X. Chen, O. Gauthier-Lafaye, J.G. Provost, C. Paranthoen and A. Fiore, 2003. Impact of intraband relaxation on the performance of a quantum-dot laser. IEEE J. Sel. Top. Quantum Electron., 9: 1308-1314.

Sugawara, M., 1995. Theory of spontaneous-emission lifetime of Wannier excitons in mesoscopic semiconductor quantum disks. Phys. Rev. B Condens. Matter., 51: 10743-10754.
Sugawara, M., 1999. Self-Assembled InGaAs/GaAs Quantum Dots. Academic Press, New York, pp: 60 .

Sugawara, M., K. Mukai, Y. Nakata and H. Ishikawa, 2000. Effect of homogeneous broadening of optical gain on lasing spectra in self-assembled InxGa1-xAs/GaAs quantum dot lasers. Phys. Rev. B, 61: 7595-7603.

Tan, C.L., Y. Wang, H.S. Djie and B.S. Ooi, 2007. Role of optical gain broadening in the broadband semiconductor quantum-dot laser. Applied Phys. Lett., 91: 061117-061119.

Tan, C.L., Y. Wang, H.S. Djie and B.S. Ooi, 2008. The role of optical gain broadening in the ultrabroadband InGaAs/GaAs interband quantum-dot laser. Comput. Mater. Sci., 44: 167-173.

Zhang, Z.Y., R.A. Hogg, P. Jin, T.L. Choi, B. Xu and Z.G. Wang, 2008. High-power quantum-dot superluminescent LED with broadband drive current insensitive emission spectra using tapered active region. IEEE Photon. Technol. Lett., 20: $782-784$. 